\title{
Implementing intelligent technical systems into smart homes by using model based systems engineering and multi-agent systems
}

\author{
J. Michael, M. Hillebrand, B. Wohlers, C. Henke, R. Dumitrescu, M. Meyer, A. Trächtler \\ Fraunhofer Project Group for Mechatronic System Design \\ Zukunftsmeile 1, 33102 Paderborn (Germany)
}

Phone number:+0049 52515465 264, e-mail: jan.michael@ipt.fraunhofer.de, michael.hillebrand@ipt.fraunhofer.de,

benedict.wohlers@ipt.fraunhofer.de, roman.dumitrescu@ipt.fraunhofer.de, christian.henke@ipt.fraunhofer.de,

matthias.meyer@ipt.fraunhofer.de, ansgar.traechtler@ipt.fraunhofer.de

\begin{abstract}
.
This paper shows a methodologically interdisciplinary approach to develop smart homes by using Model-Based Systems Engineering (MBSE) and a Multi-Agent-System (MAS) approach.

Using the methods and techniques of MBSE/MAS leads to an efficient development of a smart home and intelligent consumption of energy of the involved appliances.

At the beginning there is a specification technique used to specify an appliance. This contains analysing the requirements, evaluating the functionality of the system and choosing solution-elements. Each single system shows an intelligent, self-optimizing behaviour, which has to be up scaled to a socalled global optimum. This is reached by implementing connections between the appliances and a negotiation for available energy. Physical simulation models deliver the required energy for different processes and enable to predict the need of energy in prospective time segments. These physical models again need to be controlled by the controller models, which are also developed exemplarily in this paper. Therefore a structure of the controller is explained, which among others contains the functionality of negotiation and optimization.

This functionality is furthermore used to circumvent a defined energy-supply-bottleneck-situation.
\end{abstract}

\section{Key words}

Model-Based Systems Engineering, Multi-Agent Systems, Intelligent Technical Systems, Smart Home, Home Appliances

\section{Introduction}

Today and especially in future, the demand of renewable energy increases. The results of the United Nations Climate Change Conference can be summarized to a worldwide reduction of $\mathrm{CO} 2$ emission in the future [1]. This goal should be received by exiting the coal and atomic power production. Anyhow to provide sufficient power supplies, the renewable energies should be expanded. For instance the wind energy production has been grown worldwide by almost threefold in the past ten years [2].

Further kinds of ecologically power plants (e.g. solar power systems), also declared as decentralized or virtual power plants, have also been grown up in the last years.

One major problem of these energy sources is their supply stability and the predictability. Whereas traditional plants signify a controllable behaviour, which enables the power supplier to produce electricity relative to the demand, the virtual power plants depend on further environmental conditions.

Generating electric power with solar and wind power systems is influenced by weather conditions, which in turn can be partly predicted but not controlled.

As a consequence a more volatile power supply is expected. Prospectively there might appear situations, in which the demand for electric energy exceeds the available contingent or vice versa (e.g. during sunny/windy days). The result is an over- or underproduction of electric energy related to the market needs. Nowadays those situations already appear and the prices for electric power at the spot and energy market turn even negative [3]. Due to energy price fixe the consumer is not influenced by this price volatility so far. Perspectively the energy prices show a higher flexibility [4] so the problem of volatile energy availability is partly shifted to the consumer.

To exploit these circumstances smart systems are required which adapt their behaviour based on energy availability and daily volatile price movements. These smart systems also should be developed or private households, what this paper focusses.

What underlines the potential of this approach is the fact, that the private households are the third largest sectoral energy consumer in Germany. Therefor the effect of adaption anyway should be effectively.

Those smart systems are connected to a smart home. The term smart home describes technical processes and systems in a residential property, which enrich the quality of living, safety and enhance the efficiency in energy 
consumption [5]. The development of those smart systems requires an interdisciplinary approach. Therefor Model-Based Systems Engineering (MBSE) is used with Multi-Agent-Systems (MAS). The paradigm of MBSE ensures an efficient way for system specification and simulation whereas MAS completes MBSE to integrate communication among the smart systems. This paper is structured as follows.

At first there is a washing machine developed by using MBSE. There are requirements analysed and solution components evaluated, which fulfil the defined functionality. Furthermore the evaluated components respectively their physical behaviour is modelled and simulated to generate the power need of a washing process. The simulation is provided by a model of the control unit, which is furthermore enriched by the above described functionality of communication. This communication is developed by the MAS approach, where different roles and interactions are defined and implemented. The paper finalizes the approach by demonstrating an example of a smart home, including two appliances which negotiate available energy.

\section{Development of appliances}

To develop the appliances of a smart home there is at first each system regarded separately. This paper demonstrates a methodology by using two paradigmatic appliances; a washing machine and a voltaic cell, e.g. $\mathrm{BEV}$. These systems represent mechatronic systems and therefore this paper uses [6], a macrocycle for developing those kind of systems, and CONSENS. By using CONSENS, a design specification technique for mechatronic systems, and involving all the important stakeholder of a development process it is ensured that all the necessary requirements of the focused product are captured, what reflects the first part of the V-Model and CONSENS. CONSENS uses so-called partial-models for each step of the development. The requirements are analysed by considering all the outside influences of the system. Therefore the partial model environment is evaluated. One generic environment-model is shown in Figure 1.

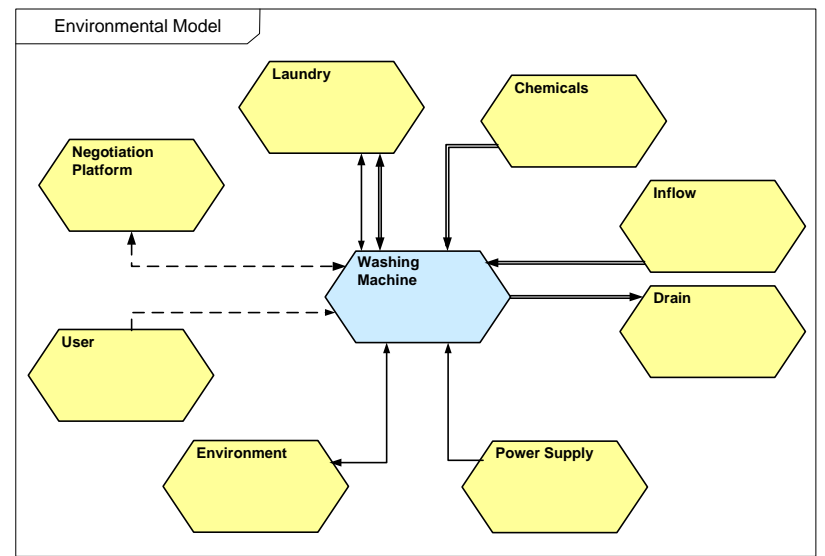

Figure 1 Environment Model Washing Machine

The result of the analysis, the requirements, is listed in the eponymous partial model. An extract of the requirements is shown in Table 1.
Table 1 Requirements Washing Machine

\begin{tabular}{|c|l|c|}
\hline Req.ID & Requirements & Priority \\
\hline $\mathbf{1}$ & Clean Laundry & A \\
\hline $\mathbf{2}$ & Access to weather data & B \\
\hline $\mathbf{3}$ & Contain User Interface & A \\
\hline $\mathbf{4}$ & Energy Label A+++ & A \\
\hline $\mathbf{5}$ & Water Security System & A \\
\hline $\mathbf{6}$ & Enable User to fill in laundry & A \\
\hline $\mathbf{7}$ & Futuristic Design & C \\
\hline $\mathbf{8}$ & Access Energy Price Data & B \\
\hline
\end{tabular}

To fulfil these requirements, CONSENS defines various functions, which are evaluated based on the requirements in form of a functional hierarchy. A washing machine needs primarily to clean laundry. Among others, one important factor for cleaning the laundry is the temperature of washing. To perform an increase of temperature, the system needs to heat the laundry. This is one of the wide range of functions, which have to be executed. Figure 2 shows an extract of the functionality of the washing machine.

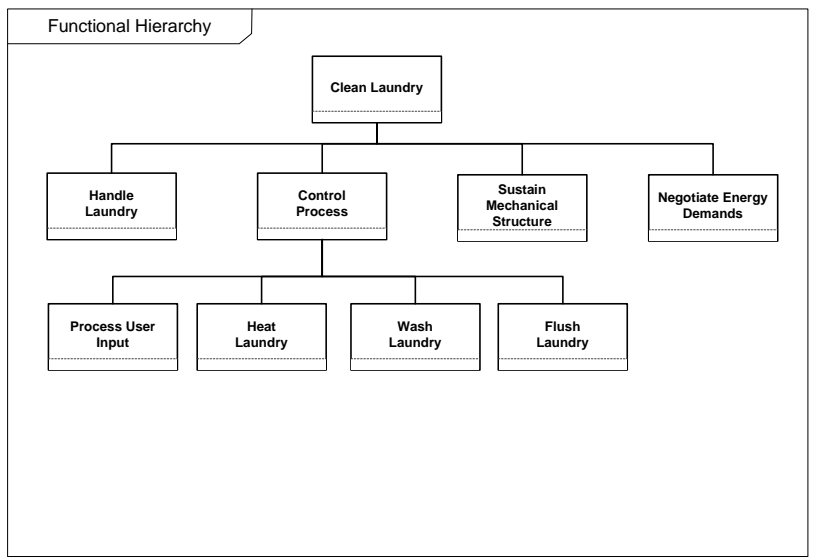

Figure 2 Functional Hierarchy Washing Machine

To perform the temperature increase there is a heating element chosen to be integrated into the system. There are further possibilities to fulfil this functionality, but within the scope of the defined requirements, there is one solution elaborated. The sum of elements of the washing machine is exemplarily subsequent listed in form of an active structure as shown in Figure 3.

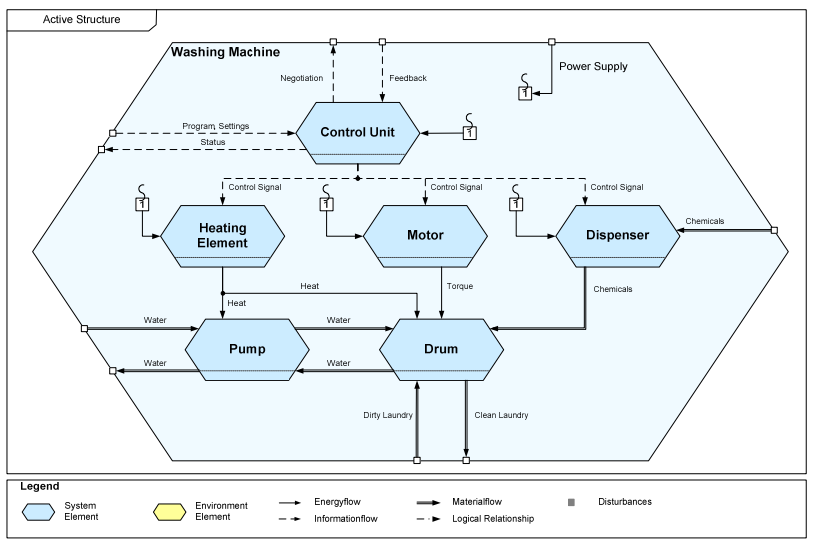

Figure 3 Active Structure Washing Machine

There are the relations between the system-elements defined. It is differentiated whether there is a logical connection, a material-, information- or an energy flow 
between the elements. Furthermore there are disturbances implemented in the active structure. As shown inFigure 3, there are listed some physical elements and a control unit to control the washing process. All these elements are afterwards modelled in form of a physical model which is simulated. It has to be defined a suitable simulation-tool, where the model is developed. In case of the washing machine there is Modelica-Dymola used for the physical components, e.g. thermodynamics. Figure 4 shows a simplified model of this heating element referring to $[7,8$.

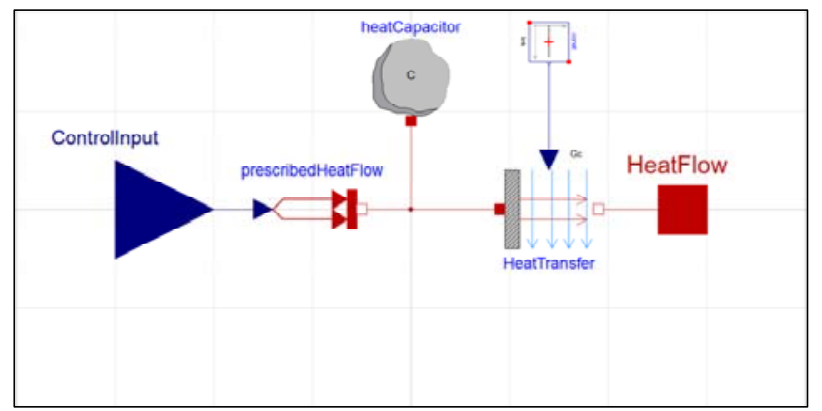

Figure 4 Physical Model Heating Element

To avoid an overhead of modelling or an insufficient model the quality of each model has to be defined early. In context of this paper the consumed energy if of main interest, to be able to predict the demand of the washing machine. Therefore the simulation model has to deliver this information. Figure 5

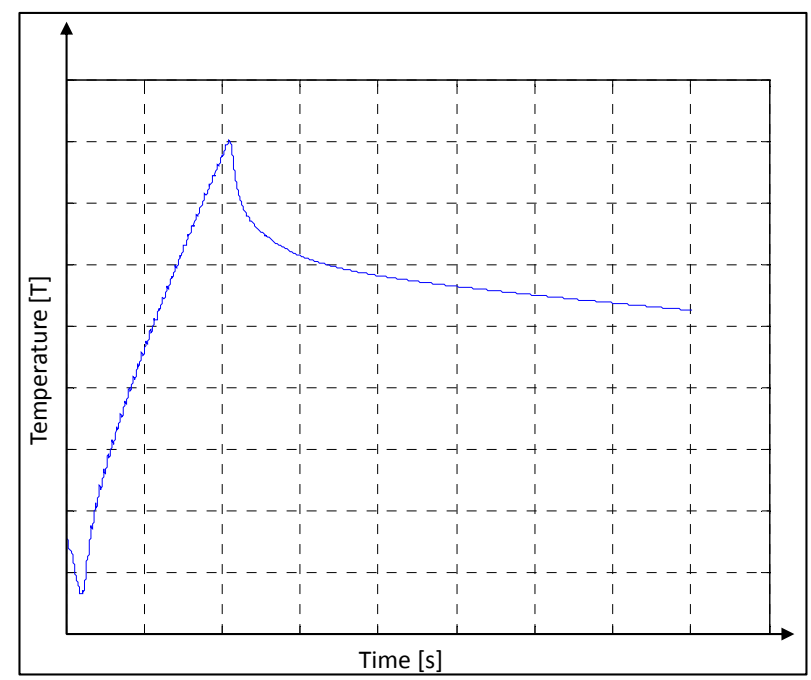

Figure 5 Simulated Temperature Signal

shows the simulation result of the fluid-temperature in the washing machine and Figure 6

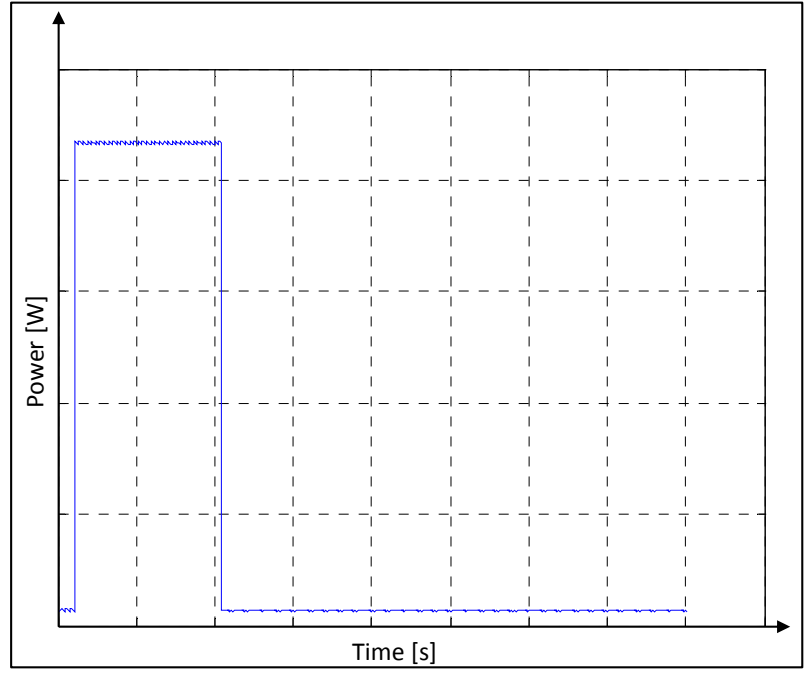

Figure 6 Simulated Power Signal

the corresponding power diagram. The subsequently following model-in-the-loop-simulations are done by implementing the physical model into Matlab-Simulink via sFunction and connecting it to the controller model.

To handle the process and perform a model-in-the-loopsimulation a control-entity is developed simultaneously with the use of Matlab Simulink Stateflow.

The architectural structure of the control unit model is implemented referring to the three-layered behavioural control from Strube [9]. [10] equally uses this approach to implement cognitive functionality into technical systems. The first layer is the so-called non-cognitive regulation; simplified controller. Related to technical systems this characterizes the simple controller. This entity has to control the physical plant continuously, e.g. trigger the actuators and sensors. The non-cognitive layer cannot process the earned sensor data such as to optimize the process, so that the coupling of stimulus-response is fixed.

Figure 7 shows the structure implemented within the use of Stateflow. As mentioned, there are some typical actuators, especially the heating element to return to the guiding thread of this paper.

The second layer of the control-architecture is the associative or reflective operator. Fundamentally this layer represents the possibility to couple designated patterns of stimulation and behaviour. This is implemented by modifying parameters and structures. Having again in mind the washing machine, the reflective operator monitors and controls the underlying noncognitive operator; it has different parametrization possibilities for the heating element and further actuators/sensors available and switches the deposited parameters of the non-cognitive operator. Some home appliances are already equipped with different parameters to generate different processes, distinguishing themselves in their energy and time demand. Another approach to flexibilize the process of home appliance is shown in [11]. The elaborated process-parameterizations are held up in data-memories, implemented in Matlab scripts, and represent one part of the reflective operator. Other parts are shown in Figure 7. 


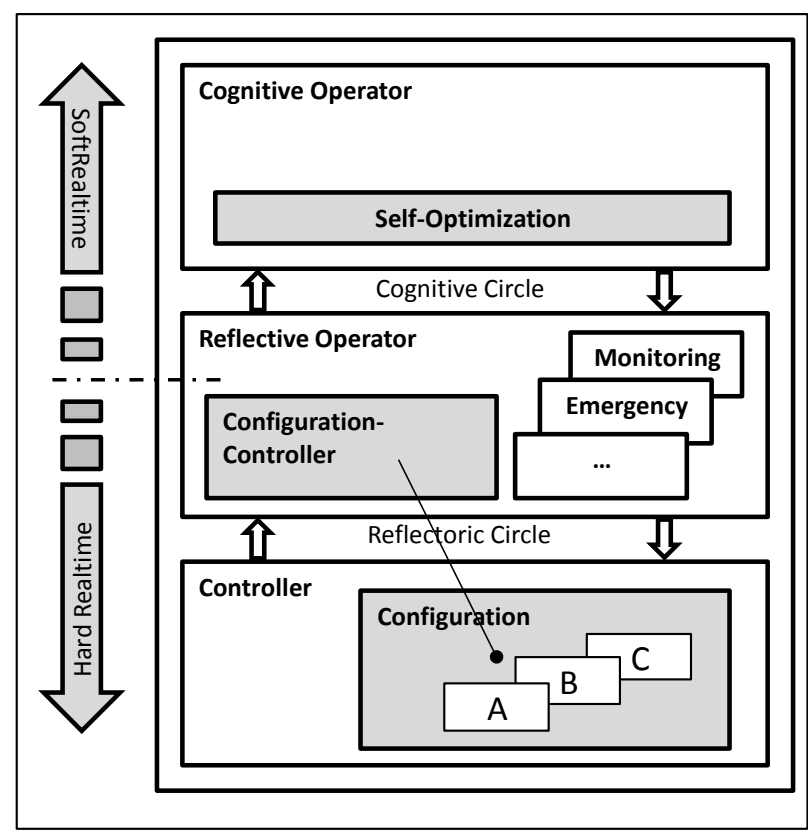

Figure 7 Structure of the Control Unit [10], modified by author

Monitoring the process and, in case of an error, triggering an emergency shutdown is the other part of the reflective operator.

The reflective operator and the controller are linked via the reflective circle. Due to the demanded real-timeconditions of the controller, the reflective operator also partially works under these conditions and processes the data with a fixed step size.

Furthermore the reflective operator can be interpreted as an interface between the controller and the cognitive operator.

The cognitive operator undertakes the functionality of information processing. This is necessary to realize a self-optimizing behaviour. With the help of diverse procedures, e.g. planning or model-based optimization, gained system-knowledge can be used to optimize the system behaviour. With the model of the washing machine and the information about changing energy prices, the process can be adapted to the prices, so that a minimum of costs is required.

The third layer is again implemented in the Matlab surrounding, because there are already efficient types of optimization-algorithms available and there can be chosen an adequate solver for each optimizationproblem, e.g. multi-objective, nonlinear or restricted problems. In case of the washing machine there are always boundary conditions like for example the user demand or further environmental conditions. Furthermore there exist vigorous nonlinearities.

All these calculations, more or less sophisticated, normally cannot be performed in real-time so that the cognitive operator works in a form of soft-real-time. In equal measure the communication with the reflective operator takes place, via the cognitive circle. As a result and as Figure 7 shows, the reflective operator is divided into a real-time and a non-real-time part, subject to the communication partner.

In the following chapter the three-layered architecture and especially the cognitive operator is addressed again.

Building up the described models enables the developer to perform model-in-the-loop simulations; to evaluate different types of heating elements in early phases of the development, without constructing a multitude of prototypes. With the help of these simulations, the requirements defined in Table 1 can already be considered, what raises the efficiency. Changes in the components have to be communicated to the requirements and vice versa.

The result of building up these models, especially in addition to intelligent control-structures, is an intelligent home appliance that can adapt its behaviour to changing environmental conditions.

\section{System of Systems}

As a result of the described course of action there are several intelligent systems, which are able to react or even to predict their behaviour. As a consequence, there may certainly appear situations, where an energy-price minimum is forecasted. The self-optimizing conduct of each appliance will naturally lead to one problem: an overload of the energy grid.

The calculated optimization results represent each a local minimum for the appliances, but in sum there will be high amplitude of energy consumption, what can lead to an overload of the grid or even to damages of electrical components. Nevertheless there is not sufficient energy to supply all the appliances.

To circumvent this problem, this paper introduces a multi-agent-system approach to ensure reconciliation between the appliances of a smart home.

For the implementation of a multi-agent-system approach, it is necessary to regard this aspect in early phases of the development. Therefore this paper enriches the CONSENS technique by the following aspects, referring to [12].

The first aspect deals with different roles of the agents in the smart grid. To demonstrate these roles, there is again the smart home focussed, which contains different appliances. Furthermore there are potentially agents, which generate energy, e.g. photovoltaic systems.

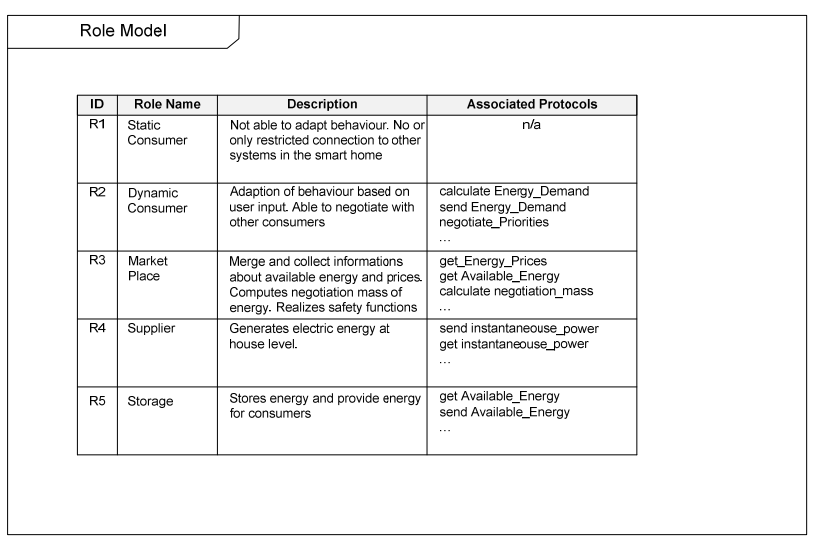

Figure 8 Role Model Smart Home

Figure 8 shows the so-called role model of different roles in a smart home. At first there are static consumers, which represent non intelligent systems, e.g. hair dryer, television. These systems are not able to adapt their behaviour/energy consumption and therefore exhibit a binary behaviour. The sum of consumption of static consumers is condensed in the residual energy and committed to the market place (described below). 
The second kind of role in the smart home is a dynamic consumer. These consumers represent exactly the functionality of the systems which were described before. Apart from adapting their energy consumption they are able to negotiate the energy. This energy is calculated as the difference between the available energy and the residual energy of the static consumers. The functionality of negotiation has to be integrated into the three-layered architecture, described in 3. Mathematically formulated the negotiation represents a further boundary condition in the optimization problem and therefore is implemented into the cognitive operator, which processes this calculation.

A further role of the system is here called the market place, which merges all the information of the remaining agents. In addition to this, the market place is connected to outer data sources, as for example weather and price forecasts; another functionality implemented into the market place is fulfilling safety functions.

The supplier, a fourth role model, describes local energy generating systems, photovoltaic systems or downscaled domestic power stations. They deliver energy, depending on e.g. weather data which can be captured from the market place.

A last kind of roles is a storage agent (e.g. Battery Electric Vehicle). This agent is able to store energy, to provide energy to consumers and receive energy from local or global suppliers.

All the role models are each defined in a role scheme, which contains a description and activities in which a role is involved. In addition to the roles there have to be some interactions proceeded. These so-called interaction models of the washing machine can be exemplarily seen in Figure 9.

\begin{tabular}{|l|l|}
\hline \multicolumn{1}{|c|}{ Interaction Model } & \\
\hline & \\
\hline $\begin{array}{l}\text { Action Name: } \\
\text { Demand required energy }\end{array}$ & Action ID: \\
\hline $\begin{array}{l}\text { Initiator: } \\
\text { R2 }\end{array}$ & $\begin{array}{l}\text { Partner: } \\
\text { R3 }\end{array}$ \\
\hline $\begin{array}{l}\text { Description: } \\
\text { When the user starts the } \\
\text { appliance, the system applies } \\
\text { the required energy for the } \\
\text { process }\end{array}$ \\
\hline
\end{tabular}

Figure 9 Interaction Model

Each interaction is given an ID, so that the interactions can be shown in the partial model environment of CONSENS and referenced in the implemented Matlab functions. The interactions concerning energy requests respectively comprise a priority, defined by additional conditions. If the user starts the washing machine and pretends to start immediately, the priority is accordingly higher than the priority of a storage, which has to be loaded. As a consequence of negotiation the process of lower priority is delayed and/or reallocated. This situation is shown in Figure 10 and Figure 11.

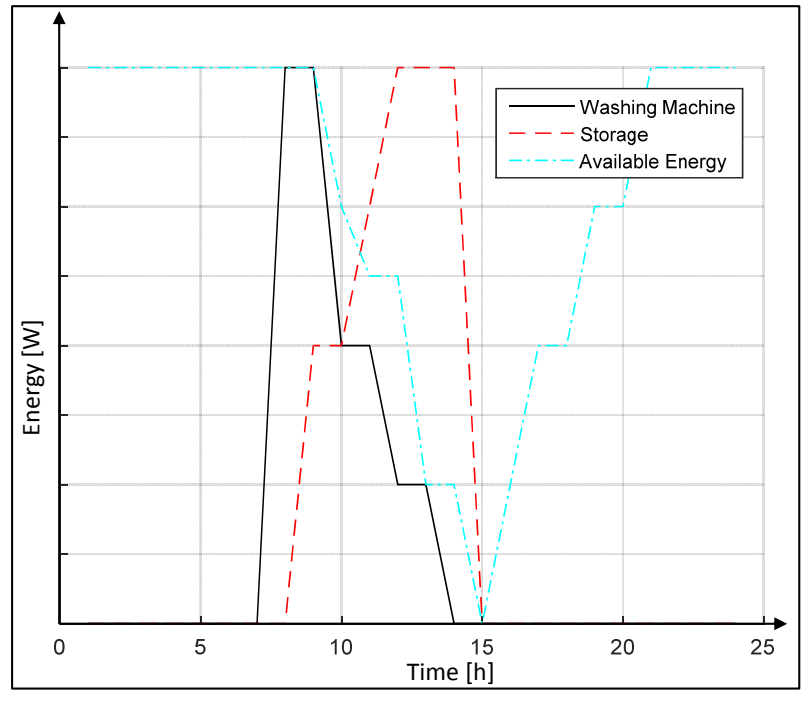

Figure 10 Requested and available Energy

There are two requests (washing machine, storage) sent to the market place, which provides the available energy, e.g. prediction of a photovoltaic system. Depending on the priorities of the two agents, the washing machine starts immediately as planned before and the storage delays and adapts the charge to the available energy. For that reason the supply bottleneck is circumvented without an outage of any system.

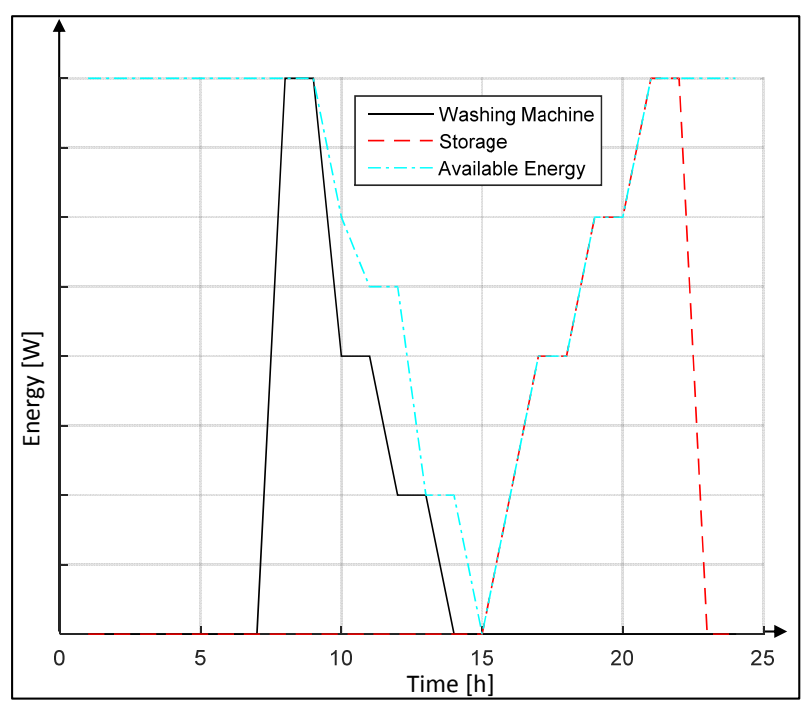

Figure 11 Delaied Requests

The interactions and the participants are visualized in the sequence diagram, shown in Figure 12.

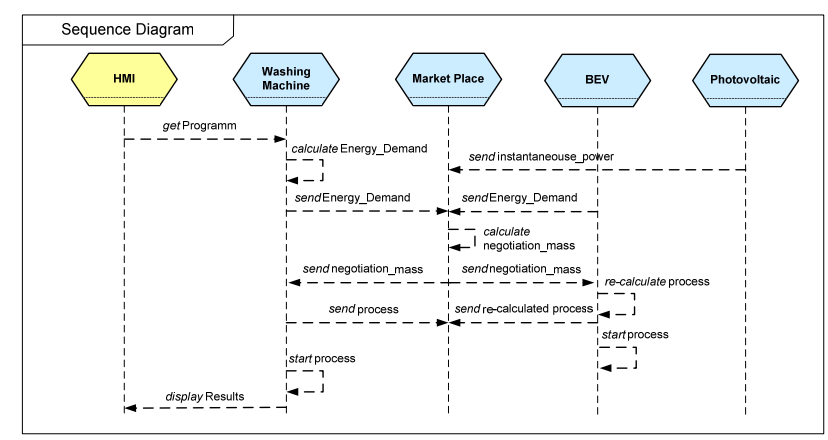

Figure 12 Sequence Diagram 


\section{Conclusion}

This paper shows a methodological interdisciplinary approach to implement intelligent technical systems into smart homes. Using MBSE and MAS is an efficient attempt to regard the overall specification of the single system, linked to other systems to negotiate the available energy of a smart home. The pursued objective is to consume energy in an intelligent way. Bypassing undersupplies and exploiting advantages of various energy prices is the focus of to be reached in a system which consists of intelligent systems.

MBSE guaranties traceability from the early system specification and is extended in this approach by the MAS attempt. In sum this leads to a system specification technique, which enables to regard connected appliances from the early specification phase to the implemented MAS.

This paper demonstrates the model-based development of smart home appliances to face actual and prospective challenges in the energy supply market.

This approach shows the development for one single smart home and can be enriched by regarding further entities. This could potentially be reached by a connection of the individual market places of each entity, to upscale the interactivity and to increase the effort of intelligent systems.

Furthermore there are some aspects to be regarded more detailed. The implementation of the negotiation and communication has to be enriched by further conditions/boundaries, to enable the inclusion of different situations and possibilities to finally continue the enhancement of efficiency.

\section{References}

[1] Federal Ministry for the Environment, Nature Conservation, Building and Nuclear Safety: Action program for climate protection. http://www.bmub.bund.de/themen/klimaenergie/klimaschutz/nationaleklimapolitik/aktionsprogramm-klimaschutz/, (02.01.2016)

[2] Federal Ministry for Economic Affairs and Energy: Energiewende direct. Issue 13/2015, https://www.bmwi-

energiewende.de/EWD/Redaktion/Newsletter/20

15/12/Meldung/infografik-infografikwindkraftkapazitaet.html, (05.01.2016)

[3] Idealo Energy Comparison: Overproduction of Energy at Pentecost strains grids and consumer. http://strom.idealo.de/news/15449-stromueberproduktion-an-pfingsten-belastet-netzeund-verbraucher/, (02.01.2016)

[4] Fraunhofer Institut for Windenergy and EnergySystem Technologies: Strommarkt Flexibilisierung- Hemmnisse und Lösungskonzepte. Study, Bochum, January 2015, ISBN-13: 978-3-920328-72-0
[5] VDE Verband der Elektrotechnik: VDERoadmap - Die deutsche Normungsroadmap Smart Home + Building. Frankfurt, November 2013

[6] VDI Verein Deutscher Ingenieure: VDI 2206 Design methodology for mechatronic systems. Guideline, Düsseldorf 2004

[7] Kruse, D.;Schweers, C.; Trächtler, A.: Methodology for a partly automated parameter identification fort the validation of multi-domain models. In: ASME International Mechanical Engineering Congress and Exposition, Montreal, 2014

[8] Kruse, D.; Trächtler, A.; Herden, R.: Modellbasierte Entwicklung eines neuartigen Heizverfahrens für Waschautomaten. In: Paderborner Workshop: „Entwurf mechatronischer Systeme “, Paderborn, 2013

[9] Strube, G.: Modeling Motivation and Action Control in Cognitive Systems. Mind Modelling, Berlin, Pabst, 1998

[10] Dumitrescu, R.: Entwicklungssystematik zur Integration kognitiver Funktionen in fortgeschrittene mechatronische Systeme. Dissertation, Paderborn, Januar 2011

[11] Michael, J.; Hillebrand, M.: Modellbasierte Mehrzieloptimierung zur Integration von Hausgeräten in Smart Grids. VDI Mechatronik Dortmund, March 2015

[12] Zambonelli, F.; Jennings, N.; Wooldridge, M.: Developing Multiagent Systems: The Gaia Methodology. ACM Transactions on Software Engineering and Methodology, Vol.12, No. 3, July 2003 